\title{
A patient with multiple arterial stenosis diagnosed with Alagille syndrome: A case report
}

\author{
Yoon Ha Lee ${ }^{1 \oplus}$, Yong Hyuk Jeon ${ }^{1 \oplus}$, Seon Hee Lim² ${ }^{2}$, Yo Han Ahn ${ }^{1,3,4}$, Sang-Yun Lee ${ }^{1 \oplus}$, Jung min Ko ${ }^{1 \oplus}$, II-Soo Ha ${ }^{1 \oplus}$, \\ and Hee Gyung Kang ${ }^{1,3,4,5, * \odot}$ \\ ${ }^{1}$ Department of Pediatrics, Seoul National University Hospital, Seoul, Korea \\ ${ }^{2}$ Department of Pediatrics, Uijeongbu Eulji Medical Center, Eulji University, Uijeongbu, Korea \\ ${ }^{3}$ Department of Pediatrics, Seoul National University College of Medicine, Seoul, Korea \\ ${ }^{4}$ Kidney Research Institute, Seoul National University Medical Research Center, Seoul, Korea \\ ${ }^{5}$ Wide River Institute of Immunology, Seoul National University, Seoul, Korea
}

\begin{abstract}
Alagille syndrome (AGS) is a rare autosomal dominant inherited disorder, with major clinical manifestations of bile duct paucity, cholestasis, cardiovascular anomaly, ophthalmic abnormalities, butterfly vertebrae, and dysmorphic facial appearance. It is caused by heterozygous mutations in JAG1 or NOTCH of the Notch signaling pathway presenting with variable phenotypic penetrance and involving multiple organ systems. The following case report describes a unique case of a 16-year-old female with AGS who presented with the primary complaint of renovascular hypertension. She had a medical history of ventricular septal defect and polycystic ovary syndrome. The patient had a dysmorphic facial appearance including frontal bossing, bulbous tip of the nose, a pointed chin with prognathism, and deeply set eyes with mild hypertelorism. Stenoocclusive changes of both renal arteries, celiac artery, lower part of the abdominal aorta, and left intracranial artery, along with absence of the left internal carotid artery were found on examination. Whole exome sequencing was performed and revealed a pathologic mutation of JAG1, leading to the diagnosis of AGS. Reverse phenotyping detected butterfly vertebrae and normal structure and function of the liver and gallbladder. While the representative symptom of AGS in most scenarios is a hepatic problem, in this case, the presenting clinical features were the vascular anomalies. Clinical manifestations of AGS are diverse, and this case demonstrates that renovascular hypertension might be in some cases a presenting symptom of AGS.
\end{abstract}

Key words: Alagille syndrome, Hypertension, JAG1, Vascular diseases.

\section{Introduction}

Alagille syndrome (AGS, Online Mendelian Inheritance in Man \# 118450) is an autosomal dominant multisystem disorder. It was first identified by Dr. Daniel Alagille in 1969, describing pa- tients with bile duct paucity and additional extrahepatic clinical features. The main clinical manifestations of AGS are infantile cholestasis with absent or reduced number of bile ducts, congenital heart anomaly (most commonly stenosis of peripheral pulmonary arteries), characteristic facies (triangular-shaped

Received: 6 September 2021, Revised: 24 September 2021, Accepted: 25 September 2021, Published: 31 December 2021

*Corresponding author: Hee Gyung Kang, M.D., Ph.D (ID https://orcid.org/0000-0001-8323-5320

Department of Pediatrics, Seoul National University Hospital, Seoul National University College of Medicine, 101 Daehak-ro, Jongno-gu, Seoul 03080, Korea.

Tel: +82-2-2072-0658, Fax: +82-2-743-3455, E-mail: kanghg@snu.ac.kr

Conflict of interest: The authors declare that they do not have any conflicts of interest.

(c) This is an open-access article distributed under the terms of the Creative Commons Attribution Non-Commercial License (http://creativecommons.org/licenses/by-nc/4.0/) which permits unrestricted non-commercial use, distribution, and reproduction in any medium, provided the original work is properly cited.

(c) Copyright 2021 by the Korean Society of Medical Genetics and Genomics 
face with a broad forehead, pointed chin, bulbous tip of the nose, deeply set eyes, and hypertelorism), butterfly vertebrae, and posterior embryotoxon [1]. Renal manifestations, including structural anomalies in the renal parenchyma and functional abnormalities, are observed in 39\% of cases, while vascular anomalies occur in 9\% to 15\% of cases [2].

AGS is suspected in patients with a combination of two or more of the following symptoms: neonatal jaundice, cardiac murmur or cyanosis, cerebral ischemic events, abdominal pain, and hypertension due to vascular anomalies. In more than $90 \%$ cases of AGS, JAG 1 gene mutations are found, followed by NOTCH2 mutations [3]. JAG 1 is a transmembrane cell-surface protein that interacts with the Notch receptors. It is essential for Notch signaling, involved in cellular differentiation, and is essential in vascular development as an inducer of vascular smooth muscle cell differentiation [4]. Pathogenic variants in the identified genes must be confirmed by molecular genetic testing to give the diagnosis of AGS.

Hypertensive children over the age of 6 years require evaluation for secondary causes of hypertension (HTN) if they do not have a family history of HTN, history of obesity, or positive physical findings according to the recent guideline [5]. Most common cause of secondary HTN is renovascular abnormalities which have been found 34\% to 79\% from previous retrospective studies. Common causes of renovascular hypertension (RVHT) include fibromuscular dysplasia, Williams syndrome, neurofibromatosis type I, tuberous sclerosis, and vasculitis [6]. Although AGS is not the main differential diagnosis for RVHT in pediatric patients because of its rare occurrence, vascular abnormalities in patients with AGS leading to RVHT have been commonly reported.

Here, we report a unique case of AGS presenting with hypertension in an adolescent female that had a significantly different clinical presentation from the conventional presentation of the syndrome finally diagnosed through nextgeneration sequencing.

\section{Case}

A 16-year-old female presented with a blood pressure (BP) of $145 / 67 \mathrm{mmHg}$ on routine examination. During evaluation for determining the cause of hypertension at a secondary hospital, renal artery stenosis was detected on abdominal computed tomography (CT) angiography. The patient was then referred to our center for further evaluation and management. She did not complain of headache, abdominal pain, or palpitations; however, she experienced intermittent weakness in the left leg with a frequency of 5 to 6 times a year, which was induced by walking for a long time and resolved immediately in seconds each time. She did not complain of nausea, dyspepsia, or jaundice. Her growth and development were normal for her age.

She was born at term with a birth weight of $2.7 \mathrm{~kg}$ without any perinatal adverse events and jaundice history. She had a ventricular septal defect (VSD), which was repaired by surgical closure at the age of one year. She started to menstruate at 12 years of age and experienced amenorrhea after the first three regular menstrual cycles. She was followed up by the gynecology department and prescribed an oral contraceptive for 1 year, under the suspicion of polycystic ovary syndrome (PCOS). She had a family history of death of her younger brother who died at the premature age of 8 years due to pulmonary atresia. Her father had diabetes mellitus, and her mother had hypothyroidism. Other familial medical histories were excluded.

On physical examination, she had frontal bossing with a prominent forehead, deep-set eyes with mild hypertelorism, a bulbous tip of the nose, and a pointed chin with prognathism. Neurological examination results were unremarkable. She had a regular pulse rate of 70 beats/min, and a grade 1-2/6 murmur was audible on the left sternal border. At the time of her first visit, her height was $160.4 \mathrm{~cm}$ (50-75 percentile), weight was $74.2 \mathrm{~kg}$ (97-99 percentile), and body mass index was 28.3 (>99 percentile). On laboratory work-up, her hemogram and blood chemistry results were found to be within the normal range. Liver function test results were normal pointing towards absence of cholestasis; the total bilirubin level was $0.5 \mathrm{mg} /$ $\mathrm{dL}$. Renal function test results were also within normal range; creatinine $0.81 \mathrm{mg} / \mathrm{dL}$ and estimated glomerular filtration rate (Chronic Kidney Disease Epidemiology Collaboration Creatinine Equation) $107.9 \mathrm{~mL} / \mathrm{min} / \mathrm{m}^{2}$. The aldosterone level was elevated to $45.6 \mathrm{ng} / \mathrm{dL}$ (normal range: $4.27-20.89 \mathrm{ng} / \mathrm{dL}$ ), whereas renin level was $2.34 \mathrm{ng} / \mathrm{mL} / \mathrm{hr}(0.32-1.84 \mathrm{ng} / \mathrm{mL} / \mathrm{hr})$. Antinuclear antibodies were present at a titer of 1:160, although there was no significant immunology-related factor that could explain the high ANA titer. Evaluations for the presence of PCOS and obesity showed hypertriglyceridemia (triglyceride level: $296 \mathrm{mg} / \mathrm{dL}$ ) and insulin resistance (insulin level: $23.7 \mu \mathrm{lU} / \mathrm{mL}$ ).

Kidney ultrasonography showed intact renal perfusion in both kidneys and delayed acceleration time in the left renal Doppler wave, indicating left renal artery stenosis. CT angiography revealed 25\% stenosis in the right renal artery, 50\% stenosis in the left renal artery, complete celiac artery occlusion, and long segmental luminal narrowing of the infrarenal abdominal aorta 
(Fig. 1). An echocardiogram was performed thereafter, which revealed a normal LV mass index of $40.4 \mathrm{~g} / \mathrm{m}^{2.7}$ and did not show any anatomical anomaly except a bilateral superior vena cava. Brain MRI revealed a steno-occlusive change in the left internal carotid artery (ICA) and left proximal anterior cerebral artery. Trans-femoral cerebral angiography showed the absence of the left ICA, but no evidence of Moyamoya disease.

Renal and abdominal aortography revealed that the celiac artery was completely occluded and reconstituted through the superior mesenteric artery (SMA). The proximal portion of the SMA also showed stenosis, and most of the flow was reconstituted through the pancreaticoduodenal arcade, and the remaining SMA flow was supplied to the collateral through the inferior mesenteric artery. Balloon angioplasty was performed on the left renal artery, and the degree of stenosis improved from 50\% to 15\% (Fig. 2). However, there was no significant decline in BP af-

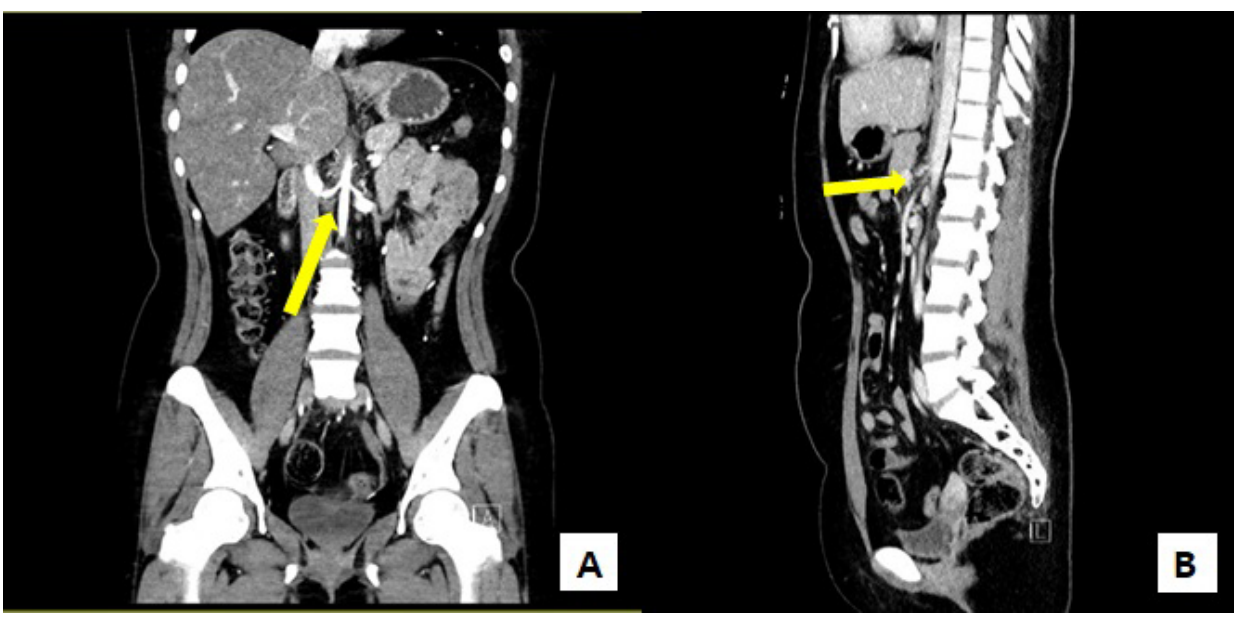

B
Fig. 1. Abdominopelvic computed tomography images. (A) The arrow indicates bilateral renal artery stenosis. (B) The arrow indicates severe stenosis of celiac artery.

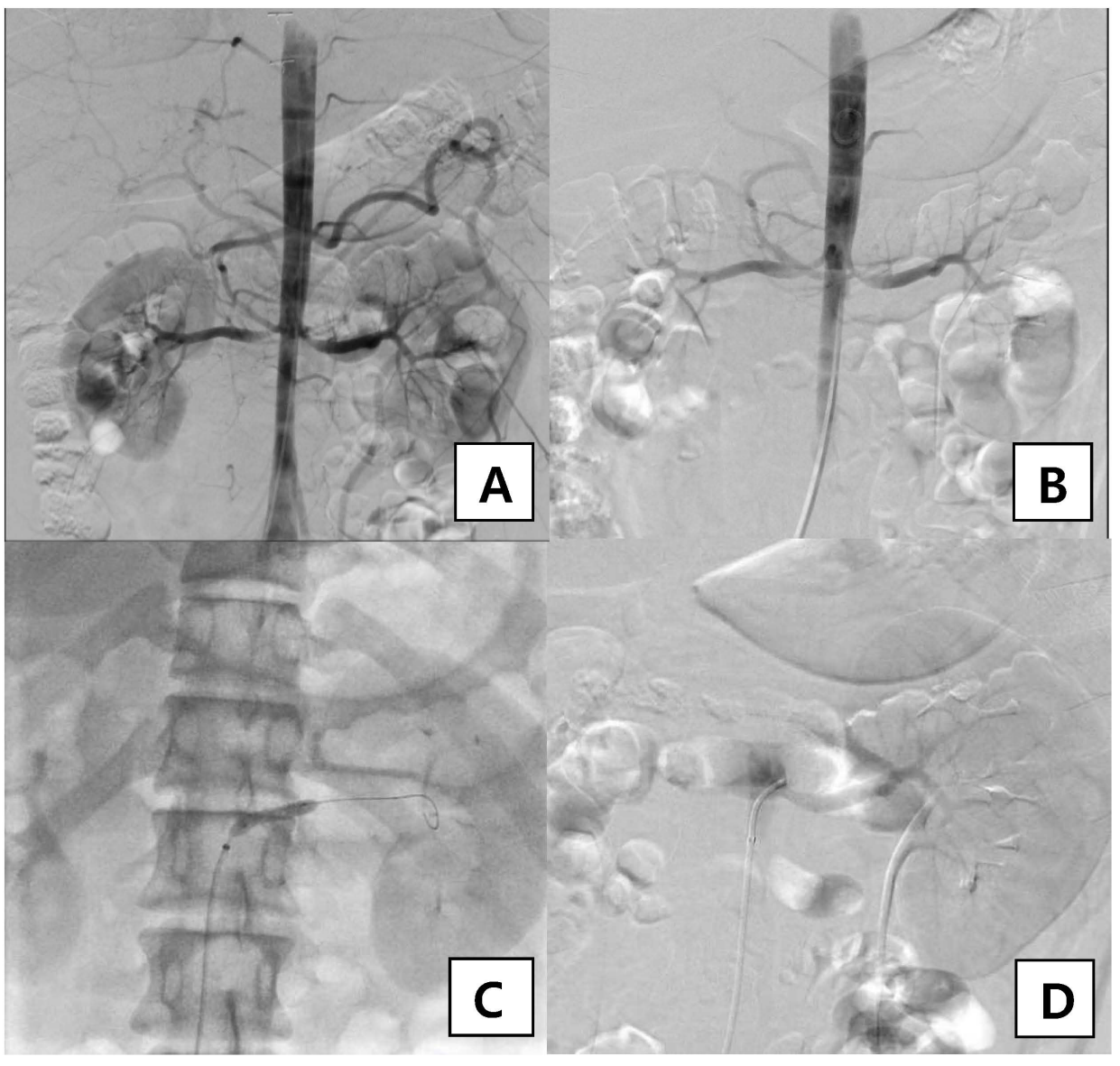

Fig. 2. Renal angiography images. (A, B) Renal angiography of the patient showing bilateral renal artery stenosis and long narrowing of infrarenal abdominal aorta. (C) Renal angioplasty was done for left renal artery stenosis. (D) Enlarged diameter of the left renal artery is after ballooning. 
ter the procedure, which may be attributed to the residual 15\% stenosis in the left renal artery and 25\% in the right renal artery.

Considering her dysmorphism, family history, and multiple vasculopathies, whole-exome sequencing was performed, which revealed a pathogenic variant, c.1899_1900delTG (p.Cys633*) in JAG1 gene. Additional evaluations were performed after diagnosis of AGS, which revealed butterfly vertebra in T7, pigmentary retinopathy in both eyes without posterior embroyotoxon, and normal structure and stiffness of liver on ultrasonography.

Nine months after the procedure, her BP remained in the range of systolic BP: 120 to $130 \mathrm{mmHg}$ and diastolic BP: 70 to $80 \mathrm{mmHg}$ with ongoing treatment using beta-blockers, angiotensin-converting enzyme inhibitors, and calcium channel inhibitors.

\section{Discussion}

Our patient in the present case was a 16-year-old female who presented with hypertension. Evaluation for the cause of the hypertension revealed severe stenosis in the both renal arteries, celiac artery, SMA, and left ICA so whole exome sequencing for multiple vascular anomaly was conducted, which led to a diagnosis of AGS. She had four manifestations suggestive of AGS: hypertension with arterial stenosis, congenital heart defect, dysmorphic facial appearance, and butterfly vertebra. Moreover, her younger brother died because of pulmonary atresia, which led us to suspect familial AGS.

In contrast to most AGS cases where the symptoms at presentation are related to congenital liver disease and heart anomalies within the first year of life [7], the clinical symptom leading to the diagnosis of AGS in our case was hypertension due to multiple vascular anomalies that afflicted the patient later in her life. There have been several case reports on hypertension occurring after adolescence, mainly due to stenosis in a single artery. According to Yucel et al. (2010) [8], a woman presented with hypertension at 38 years of age with renovascular stenosis. The patient also had a history of pulmonary artery stenosis and neonatal jaundice and was therefore finally diagnosed with AGS. In another case series, three members of a family aged 12,50, and 62 respectively were diagnosed with AGS during evaluation for high BP. All of them had renal artery stenosis, and the 12-year-old patient had multiple arterial stenoses, including the left common iliac vein, external iliac artery, and left subclavian artery [9]. To the best of our knowledge, there are no reports to date on AGS diagnosed with multiple arteriopathies in adolescents in Korea.

Vascular anomalies of AGS have been reported in 9\% to $15 \%$ of cases $[2,10]$. In a study, among 25 AGS patients who underwent abdominal imaging before liver transplantation, 12 patients (48\%) had celiac artery stenosis, 2 patients had renal artery stenosis, and 2 patients had SMA stenosis. One patient had multiple arteriopathies, including the renal artery, SMA, and celiac artery [11]. Vascular anomalies in AGS mostly involve intracranial arteries and less frequently the renal artery or aorta [12]. Although the frequency of vascular anomalies is low, morbidity and mortality from noncardiac vasculopathy have been reported in $34 \%$ of the patients $[1,13]$.

Interestingly, such multiple vasculopathies have not been reported in any of the previous case reports on AGS with the same pathogenic variant (c.1899_1900delTG, p.Cys633*) in JAG 1 gene $[14,15]$. A previously reported case of the same variant had biliary atresia, congenital heart disease, skeletal anomaly, maculopathy, and peculiar facies except vasculopathy [15]. Therefore, it can be concluded that the genotype-phenotype correlation is not strong in AGS. Recently, several studies have suggested that secondary modifier genes of JAG1 protein, such as POGLUT1 and THBS2 affect JAG1 protein expression [14].

In addition to vasculopathy of AGS, our case also had coexisting PCOS, although its association with AGS has not been reported in the past. PCOS has proven association with cardiovascular disease risk, elevation in coronary artery calcium scores and carotid intima-media thickening [16]. The patient in the present case had dyslipidemia, which may have aggravated the vasculopathy due to atherosclerosis. In addition, the patient also had PCOS, which might have further contributed to the condition. Thus, this patient was at a higher risk of complications arising from vasculopathy, mandating close monitoring and management.

AGS is an autosomal dominant inherited disease, but $60 \%$ of patients have de novo mutation. In this case, the younger brother suffered from severe pulmonary atresia pathogenic mutation, which is supposed to be passed down from one of the parents. Her father had diabetes mellitus, and her mother had hypothyroidism; however, they haven't been diagnosed with any diseases related to AGS. This genetic phenomenon can be supported by germline mutations, whose frequency is $8 \%$ in AGS [1], but more detailed history taking and familial genetic evaluation are necessary. This study is constrained by the limitation that no familial genetic testing was done and unfortunately history taking from the grandparents for each family was omitted.

Currently, there is no curative treatment for AGS. Therefore, 
the mainstay of treatment is symptomatic relief. Nonetheless, the prognosis of AGS has improved over time with advances in the surgical and medical management of the related problems, such as congenital heart disease and bile duct paucity. However, the major challenge is that vasculopathy is often found by chance or in acute status such as hemorrhage or infarction during late adolescence or adulthood [13]. Therefore, screening for non-cardiac vasculopathy is necessary for patients with AGS. In addition, vasculopathy can be progressive and resistant to medical treatment necessitating regular imaging examinations. Our patient had a high risk of left-side cerebral infarction, progressive abdominal aortic vasculopathy, and renal artery stenosis, requiring regular monitoring for disease progression.

In conclusion, we presented a case of an adolescent patient with a medical history of VSD and PCOS who was incidentally found to have severe hypertension. Upon thorough evaluation it was revealed that she had secondary hypertension with narrowing of a number of arteries. The underlying cause for these findings was revealed to be AGS. This case is unique with respect to the fact that its presentation was quite different from the known typical disease course of AGS.

\section{Acknowledgements}

This work was supported by the National Research Foundation of Korea (NRF) grant funded by the Korea government (MSIT) (No. NRF-2020R1A2C1100974). We are also grateful to the patient and her parents for providing consent for this study.

\section{Authors' Contributions}

Conception and design: HGK. Acquisition of data: YHL, YHJ. Analysis and interpretation of data: none. Drafting the article: YHL, YHJ. Critical revision of the article: SHL, YHA, SYL, JK, ISH, HGK. Final approval of the version to be published: all authors.

\section{References}

1. Turnpenny PD, Ellard S. Alagille syndrome: pathogenesis, diagnosis and management. Eur J Hum Genet 2012;20:251-7.

2. Kamath BM, Spinner NB, Emerick KM, Chudley AE, Booth C, Piccoli DA, et al. Vascular anomalies in Alagille syndrome: a significant cause of morbidity and mortality. Circulation 2004;109:1354-8.

3. Spinner NB, Gilbert MA, Loomes KM, Krantz ID. Alagille syndrome. In: Adam MP, Ardinger HH, Pagon RA, Wallace SE, Bean DH, Mirzaa G, eds. GeneReviews ${ }^{\circledR}$. Seattle (WA): University of Washington, Seattle, 1993-2021.

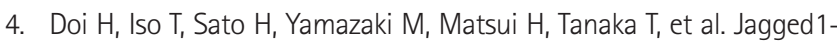
selective notch signaling induces smooth muscle differentiation via a RBP-Jkappa-dependent pathway. J Biol Chem 2006;281:28555-64.

5. Flynn JT, Kaelber DC, Baker-Smith CM, Blowey D, Carroll AE, Daniels SR, et al.; Subcommittee on Screening and Management of High Blood Pressure in Children. Clinical practice guideline for screening and management of high blood pressure in children and adolescents. Pediatrics 2017;140:e20171904.

6. Patel PA, Cahill AM. Renovascular hypertension in children. CVIR Endovasc 2021;4:10.

7. Kamath BM, Baker $A$, Houwen $R_{1}$ Todorova L, Kerkar N. Systematic review: the epidemiology, natural history, and burden of Alagille syndrome. J Pediatr Gastroenterol Nutr 2018;67:148-56.

8. Yucel $H_{1}$ Hoorntje SJ, Bravenboer B. Renal abnormalities in a family with Alagille syndrome. Neth J Med 2010;68:38-9.

9. Shrivastava R, Williams $A$, Mikhail $A$, Roberts D, Richards M, Aithal V. An unusual cause of hypertension and renal failure: a case series of a family with Alagille syndrome. Nephrol Dial Transplant 2010;25:15016.

10. Emerick KM, Rand EB, Goldmuntz E, Krantz ID, Spinner NB, Piccoli DA. Features of Alagille syndrome in 92 patients: frequency and relation to prognosis. Hepatology 1999;29:822-9.

11. Kohaut J, Pommier R, Guerin F, Pariente $D_{\text {, Jacquemin }}$, Martelli $H_{\text {, et }}$ al. Abdominal arterial anomalies in children with Alagille syndrome: surgical aspects and outcomes of liver transplantation. J Pediatr Gastroenterol Nutr 2017;64:888-91.

12. Raas-Rothschild A, Shteyer E, Lerer I, Nir A, Granot E, Rein AJ. Jagged1 gene mutation for abdominal coarctation of the aorta in Alagille syndrome. Am J Med Genet 2002;112:75-8.

13. Sanada $Y$, Naya I, Katano T, Hirata $Y$, Yamada $N$, Okada N, et al. Visceral artery anomalies in patients with Alagille syndrome. Pediatr Transplant 2019;23:e13352.

14. Gilbert MA, Bauer RC, Rajagopalan R, Grochowski CM, Chao G, McEldrew $D$, et al. Alagille syndrome mutation update: comprehensive overview of JAG1 and NOTCH2 mutation frequencies and insight into missense variant classification. Hum Mutat 2019;40:2197-220.

15. Dědič $T$, Jirsa $M$, Keil R, Rygl $M$, Šnajdauf J, Kotalová R. Alagille syndrome mimicking biliary atresia in early infancy. PLoS One 2015;10:e0143939.

16. Osibogun 0, Ogunmoroti O, Michos ED. Polycystic ovary syndrome and cardiometabolic risk: opportunities for cardiovascular disease prevention. Trends Cardiovasc Med 2020;30:399-404. 